\title{
4. Reinigungsstufe auf Kläranlagen zur weitergehenden Behandlung kommunaler Abwässer
}

\author{
Heidemarie Schaar · Norbert Kreuzinger
}

Online publiziert: 21. Juni 2017

(c) Der/die Autor(en) 2017. Dieser Artikel ist eine Open-Access-Publikation.

\begin{abstract}
Zusammenfassung Über die letzten Jahre entwickelten sich die Überlegungen zu einer über den derzeitigen Stand der Technik hinausgehenden Abwasserreinigung von reinen forschungsorientierten Ansätzen hin zu einer großtechnischen Implementierung, sodass es heute für die unterschiedlichen verfahrenstechnischen Varianten einer weitergehenden Abwasserreinigung zur Entfernung von organischen Spurenstoffe bereits großtechnische Umsetzungen gibt. Vor allem in der Schweiz hat aufgrund der gesetzlichen Rahmenbedingungen die Umsetzung einer 4. Reinigungsstufe bereits begonnen, wenngleich auch noch eine Reihe von Detailfragestellungen für eine technische Implementierung offen ist. Dieser Beitrag fasst die heute anerkannten Optionen für Verfahren zur weitergehenden Abwasserreinigung zusammen und ergänzt sie durch Erkenntnisse aus einem vom BMLFUW geförderten nationalen Forschungsprojekt "KomOzAk“, das dazu beiträgt, die Umsetzung einer 4. Reinigungsstufe technisch und betrieblich zu optimieren.
\end{abstract}

\section{Advanced treatment steps for a further removal of organic trace pollutants from municipal wastewater}

\footnotetext{
Abstract Over the last years, considerations for an implementation of advanced treatment steps for the removal of organic trace pollutants shifted from a rather research oriented approach focusing on the removal potential of considered technologies towards a fullscale implementation. As an according legislation is put into place, especially in Switzerland full-scale units

Dr. H. Schaar $(\varangle) \cdot$ Dr. N. Kreuzinger Institut für Wassergüte, Ressourcenmanagement und Abfallwirtschaft, Technische Universität Wien, Karlsplatz 13/226-1, 1040 Wien, Österreich hschaar@iwag.tuwien.ac.at
}

comprising different technologies and combinations of technologies already are realized and in place. This paper gives an overview on treatment technologies that proved to be suitable for tackling the topic of further removal of organic trace pollutants from municipal wastewater. This overview is supplemented by results of an Austrian research project looking into details on technical and operational optimization of ozonation and activated carbon application.

\section{Herausforderungen an die Abwasserreinigung}

Die Anforderungen an die Reinigungsleistung von Kläranlagen lassen sich historisch gesehen direkt aus den Anforderungen des Gewässerschutzes ableiten. Der hohe Standard der Abwasserreinigung in Österreich hat wesentlich zur Verringerung der Gewässerbelastungen mit Kohlenstoff, Stickstoff und Phosphor und damit zum heutigen guten Zustand der Gewässer beigetragen. Heutzutage resultieren an Kläranlagen gestellte Anforderungen jedoch nicht mehr ausschließlich aus Aspekten des Gewässerschutzes. Themen wie Energieeffizienz und eine Betrachtung des Abwassers als wertvolle Ressource im Sinne des NEW-Ansatzes (NährstoffEnergie-Wasser-Recycling aus Abwasser) sind heute ebenso wichtig wie das traditionelle Vorsorgeprinzip und die weitergehende Entfernung anthropogener Verunreinigungen, um potenziell adversen Effekten im Gewässer entgegenzuwirken. Sowohl der Reuse-Aspekt, als auch der Schutz des Gewässerökosystems und der Schutz von Trinkwasserressourcen sind die Ausgangspunkte für die Diskussionen der Implementierung einer 4. Reinigungsstufe auf Kläranlagen.

Seit den frühen 2000er-Jahren beschäftigt sich die Wissenschaft mit verfahrenstechnischen Möglichkeiten zur Entfernung organischer Spurenstoffe aus dem Abwasser. Ausgangspunkt davon war die Thematik hormonell wirksamer Substanzen und ihre Auswirkungen auf Gewässerorganismen, wie sie z. B. in Großbritannien oder anderen Ländern mit damals traditionell nur Kohlenstoff entfernenden Kläranlagen beobachtet wurden. Ausgehend von den unterschiedlichen Standards der Abwasserreinigung wurde zudem der Frage nachgegangen, inwieweit bestehende Kläranlagen nach dem Stand der Technik diese Substanzen bereits entfernen. Die in Österreich umgesetzten Schwachlastanlagen mit Nitrifikation und Denitrifikation entfernen natürliche weibliche Sexualhormone wie Estradiol sowie synthetische Hormone wie Ethinylestradiol zu etwa $90 \%$. Die emittierten Rest-Konzentrationen liegen dennoch über den definierten Wirkkonzentrationen. Neben hormonell wirksamen Substanzen finden sich zahlreiche andere organische Spurenstoffe, wie im Haushalt eingesetzte Industriechemikalien oder pharmazeutische Wirkstoffe im Ablauf von Kläranlagen. Teilweise entziehen sich organische Spurenstoffe den klassischen Abbaumechanismen und verlassen die Kläranlagen gegenüber dem Zulauf in unveränderten Konzentrationen.

\section{Verfahrenstechnische Möglichkeiten für eine 4. Reinigungsstufe zur weitergehenden Entfernung organischer Spurenstoffe aus kommunalem Abwasser}

Die Forschung konzentrierte sich zunächst auf das Entfernungspotenzial von Verfahren, die aus der Trinkwasseraufbereitung bekannt sind, wobei Membransysteme, Ozonung und Aktivkohlefiltration im Mittelpunkt der Betrachtungen standen. Eine hohe zeitliche Variabilität, Schwankungen der Abwasserzusammensetzung und Matrix aus saisonalen Gründen sowie Verdünnung bei Regenereignissen führen jedoch zu einer starken qualitativen und quantitativen Dynamik, sodass 
PAK
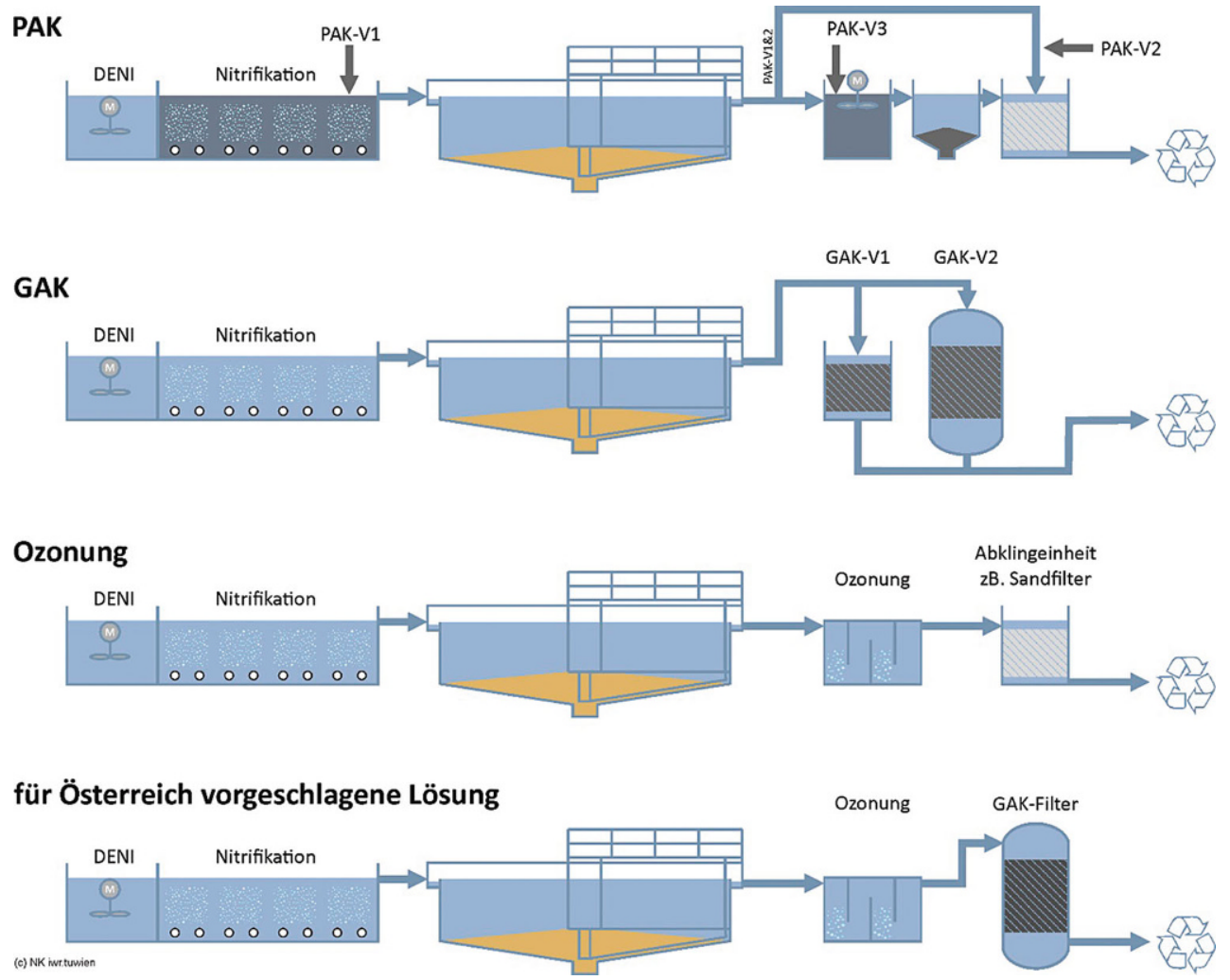

Abb. 1 Varianten einer 4. Reinigungsstufe auf Kläranlagen zur weitergehenden Behandlung kommunaler Abwässer

eine direkte Umlegung der Verfahren aus dem Trinkwassersektor nicht möglich ist.

Bei den untersuchten Verfahren zeigte sich, dass die in der Abwasserreinigung eingesetzten Membranverfahren (Mikrofiltration) nicht geeignet sind, organische Spurenstoffe zurückzuhalten, sodass sich die Forschung in den letzten Jahren auf oxidative (Ozonung und AOP-Advanced Oxidation Processes) sowie adsorptive Verfahren (Aktivkohleanwendung) fokussierte. Bei den oxidativen Verfahren kommt es $\mathrm{zu}$ einer Zerstörung der Zielsubstanzen, wogegen sich bei den adsorptiven Verfahren die Zielsubstanzen an die Aktivkohle anlagern und so aus dem Wasserstrom entfernt werden. Heute werden die in Abb. 1 skizzierten Verfahren für großtechnische Umsetzungen der 4. Reinigungsstufe herangezogen, wobei vermehrt der biologischen Stufe nachgeschaltete Verfahren zum Einsatz kommen.

\subsection{Pulveraktivkohle - PAK}

Bei der PAK-Anwendung wird dem Reinigungsprozess pulverförmige Aktivkohle (Partikelgröße im $\mu \mathrm{m}$ Bereich) zugegeben. Die Zugabe der Aktivkohle kann etwa direkt im Belebungsbecken erfolgen (PAK-V1). Die Kohle wird dabei in den Belebtschlamm eingelagert und weitgehend mit dem Überschussschlamm entfernt. Auf Grund der geringen Partikelgröße der PAK erfolgt jedoch kein quantitatives Absetzen der Kohle im Nachklärbecken und es muss durch einen nachgeschalteten Filter (Sand- bzw. Anthrazitfilter) verhindert werden, dass mit Spurenstoffen beladene Kohle ins Gewässer ausgetragen wird. Die Zugabe der AK kann auch unmittelbar vor diesem Filter erfolgen (PAK-V2) oder in einen separaten Kontaktor mit anschließender Sedimentation (PAK-V3).

\subsection{Granuläre Aktivkohle - GAK}

Die Anwendung von granulärer Aktivkohle (Partikelgröße im mm Bereich) erfolgt als Filtrationsstufe, indem das zu behandelnde Rohwasser durch einen mit GAK gefüllten Filter fließt. Die Filter können als offene Filter (GAK-V1) oder geschlossene Druckfilter (GAK-V2) ausgeführt sein und orientieren sich am Konzept, wie es auch in der Trinkwasseraufbereitung zum Einsatz kommt. Vorhandene, nachgeschaltete Sandfilter können relativ einfach auf einen
Betrieb mit GAK umgerüstet werden. Die GAK-Filter werden rückgespült und benötigen keine nachgeschaltete Stufe zum Rückhalt von feinen Kohlepartikeln, wie dies bei der PAK-Anwendung der Fall ist.

\subsection{Ozonung}

Bei der Ozonung wird vor Ort aus Sauerstoff gasförmiges Ozon hergestellt, das über ein geeignetes Eintragssystem (Injektoren oder Diffusoren) in den Abwasserstrom eingetragen wird. Dabei wirken sowohl Ozon als auch bei Ozonreaktionen entstehende OH-Radikale und zerstören die organischen Spurenstoffe. Je nach Abwassermatrix können bei diesem Vorgang auch unspezifische Nebenprodukte entstehen, die gemeinsam mit eventuell vorhandenem Restozon in einer abschließenden Einheit entfernt werden sollten. Dafür eignen sich etwa ein Sand-, Hydroanthrazit oder Aktivkohlefilter. Bei der Ozonung kommt es im Gegensatz zur Aktivkohleadsorption auch zu einer Abtötung von Bakterien und somit zu einer Teildesinfektion des Abwassers um mehrere Zehnerpotenzen.

Beide heute als geeignet betrachteten Verfahren (Ozonung und Aktivkoh- 


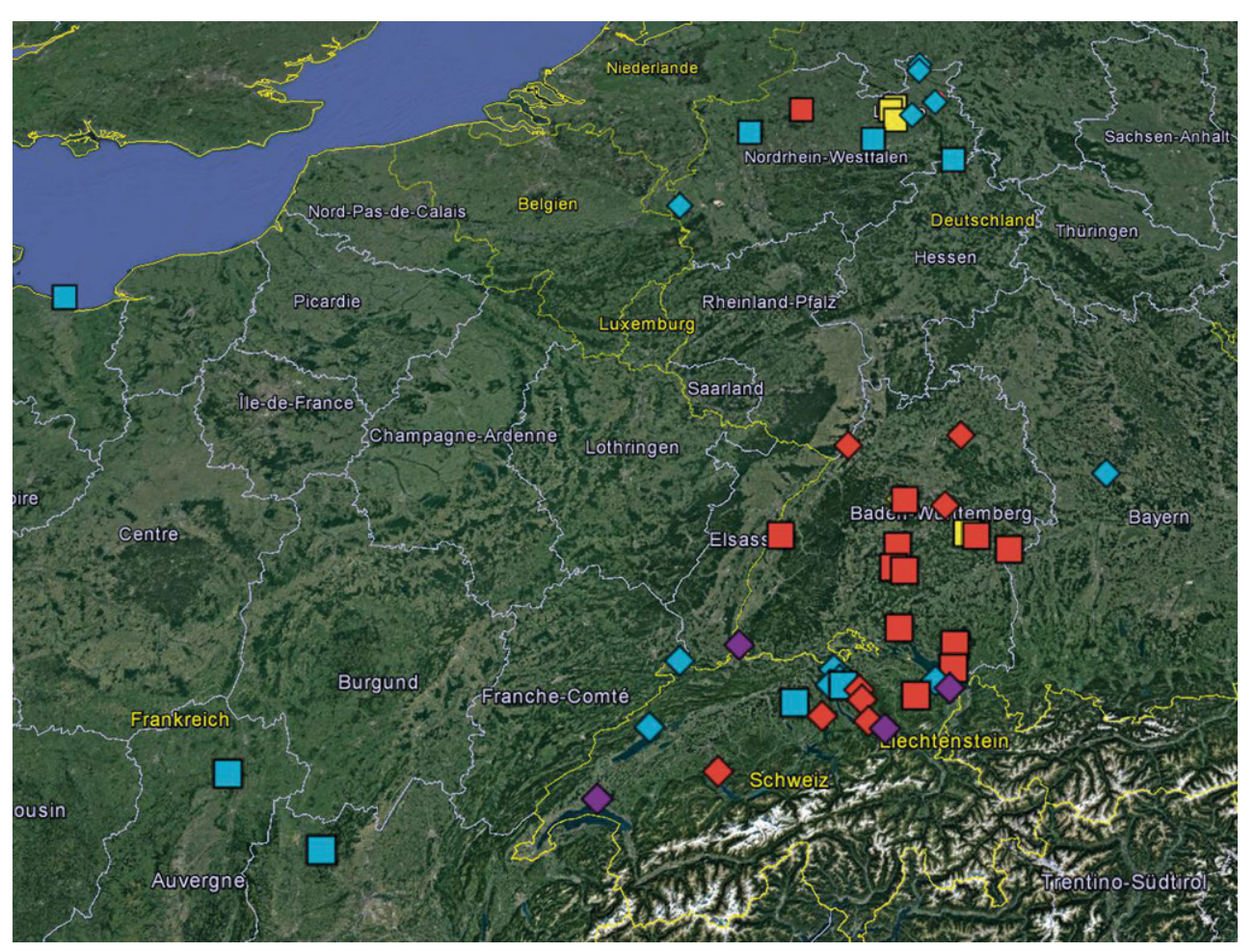

Abb. 2 Übersichtskarte Spurenstoffelimination auf Kläranlagen; Legende: türkis ... Ozonung, rot ... PAK, gelb ... GAK, violett ... Verfahrenskombination; Rechteck ... in Betrieb; Raute ... in Planung/Bau (ergänzt und modifiziert aus: VSA-Plattform „Verfahrenstechnik Mikroverunreinigungen“ https://www.micropoll.ch/anlagen-projekte/Übersichtskarte/)

leanwendung) können aufgrund deren physikalisch-chemischen Eigenschaften für sich alleine nicht alle organischen Spurenstoffe entfernen. Beide weisen Vor- und Nachteile auf, sodass keine der beiden Technologien a priori $\mathrm{zu}$ präferieren ist. $\mathrm{Zu}$ den verfahrensimmanenten Aspekten kommen zusätzlich länderspezifische Aspekte zum Tragen, die bei einer Verfahrensauswahl eine wesentliche Rolle spielen. Im Projekt KomOzAk „Weitergehende Reinigung kommunaler Abwässer mit Ozon sowie Aktivkohle für die Entfernung organischer Spurenstoffe" wurden Verfahrensvarianten und -kombinationen näher untersucht, welche die in Österreich gegebenen Rahmenbedingungen auf Kläranlagen berücksichtigen. Für Österreich wird eine aus Ozonung und nachgeschalteter, biologisch aktivierter granulärer Aktivkohlefiltration bestehende Verfahrenskombination als sinnvollste Lösung erachtet. Diese Kombination (siehe Abb. 1) kompensiert die Nachteile der Einzelverfahren und stellt zudem ein Multibarrierensystem dar, das neben einer Maximierung der Spurenstoffentfernung vor allem in Hinblick auf die Investitionssicherheit für die Betreiber als nachhaltige Lösung erachtet wird.

\section{Realisierte Anlagen im DACH-Bereich und Rahmenbedingungen}

Mit der Revision der Gewässerschutzverordnung, die am 1. Jänner 2016 in Kraft trat, ist die Schweiz bei der Implementierung einer 4 . Reinigungsstufe zur gezielten weitergehenden Entfernung organischer Spurenstoffe weltweit am weitesten fortgeschritten. Seit Anfang 2014 ist in der Schweiz auf der Kläranlage Neugut (Zürich) mit einer Ozonung die erste großtechnische 4. Reinigungsstufe in Betrieb. Auch in Deutschland (v. a. Baden-Württemberg und in Nordrhein-Westfalen) gibt es bereits großtechnische Umsetzungen und zahlreiche weitere Planungen. Abb. 2 zeigt die mit Stand Frühjahr 2017 in Betrieb bzw. in Umsetzung befindlichen 4. Reinigungsstufen in der DACHRegion.

In diesem Zusammenhang ist es wichtig, auf länderspezifische Rahmenbedingungen hinzuweisen, die - im Gegensatz zu Österreich - in der Schweiz und in Deutschland gegeben sind und sich auf die Implementierung einer 4. Reinigungsstufe auswirken. In beiden Nachbarländern sind nachgeschaltete Sandfilter weit verbreitet, die historisch für einen Partikelrückhalt geplant wurden. Obwohl sich diese oftmals nicht in Betrieb befinden, existieren die Bauwerke dennoch und eine Umrüstung in eine 4 . Reinigungsstufe ist vergleichsweise einfach möglich, zumal auch die damit verbundenen hydraulischen Aspekte auf den Kläranlagen bereits berücksichtigt sind. In Deutschland kommt zusätzlich der Aspekt der Abwasserabgabe zum Tragen. Diese ist vom Betreiber abzuführen und fußt wesentlich auf den emittierten CSBFrachten. Durch den Einsatz von Aktivkohle als 4. Reinigungsstufe wird nun auch an und für sich unbedenklicher, weil nicht mehr biologisch abbaubarer CSB (z. B. Huminstoffe) entfernt, womit sich für den Betreiber die Ausgaben für die Abwasserabgabe deutlich reduzieren und diese Einsparungen für die 4. Reinigungsstufe gegengerechnet werden können. Dieser Aspekt tritt bei der Ozonung nicht auf, sodass in Deutschland die Aktivkohle einen gewissen monetären Vorteil genießt. 


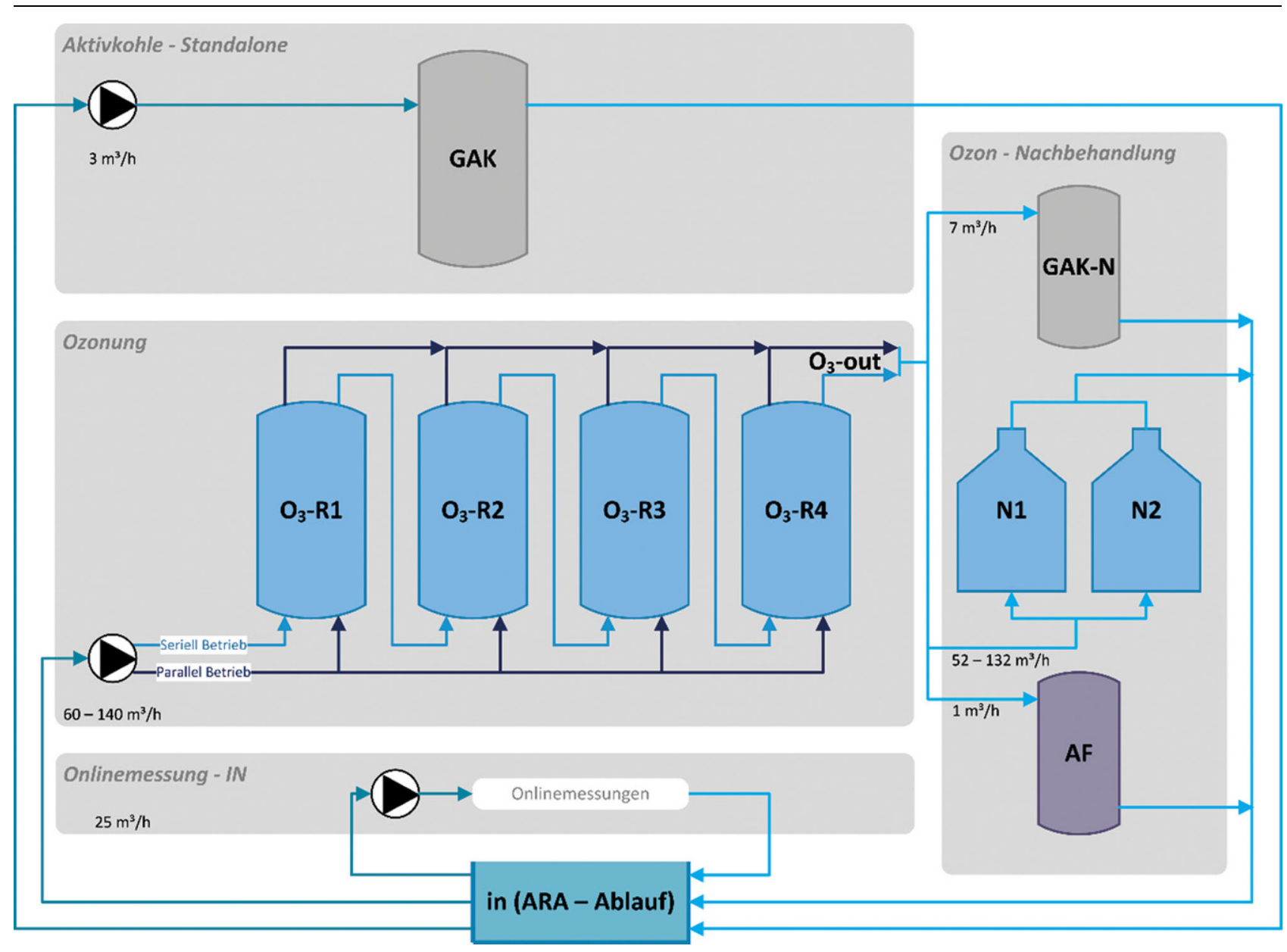

Abb. 3 Übersicht über die KomOzAk Anlage

4 Ergebnisse aus dem österreichischen Forschungsprojekt „KomOzAk“

Im Rahmen des vom BMLFUW mitfinanzierten Forschungsprojektes „KomOzAk“ wurde eine Versuchsanlage zur weitergehenden Abwasserreinigung mittels Ozon und granulärer Aktivkohle untersucht (Abb. 3), woraus sich folgende Erkenntnisse ableiten lassen.

Wie in vorangegangenen Studien erwies sich die Kläranlagenablaufozonung im untersuchten Standardbetrieb (spezifische Ozondosis von $0,7 \mathrm{~g} \mathrm{O}_{3} / \mathrm{g}$ DOC $_{\text {Ablauf }}$ als robustes und ausfallsicheres Verfahren. Für die Dimensionierung einer Ozonung stellt neben der hydraulischen v.a. die organische Belastung des Abwassers einen wesentlichen Parameter dar. Die organische Matrix hat den größten Einfluss auf die Ozonzehrung, da sie zahlreiche ozonreaktive Verbindungen enthält. Dies impliziert, dass Ozon bezogen auf die organische Hintergrundmatrix des Ab- wassers, gemessen als DOC, dosiert werden sollte.

Das Entfernungspotenzial von Spurenstoffen hängt grob gesprochen von zwei Faktoren ab: einerseits von der Reaktivität der Spurenstoffe mit Ozon und mit OH-Radikalen und andererseits von der Ozon- bzw. OH-Radikalexposition, d.h. dem Produkt aus Konzentration und Expositionszeit. Letzteres wird wesentlich von der DOC-Konzentration und -Qualität im Kläranlagenablauf beeinflusst, was nochmals die Relevanz der Organik unterstreicht. Ab einer spezifischen Ozondosis von etwa $0,4 \mathrm{~g} \mathrm{O}_{3} / \mathrm{g}$ DOC werden ozonaffine Indikatorsubstanzen, wie z. B. Carbamazepin oder Diclofenac bis unter die Nachweisgrenzen entfernt. Bei moderat mit Ozon reagierenden Substanzen zeigt sich ein deutlicher Zusammenhang zwischen Spurenstoffentfernung und spezifischer Ozondosis, der in Abhängigkeit der Reaktionskonstanten mit Ozon variierte. So wird bei $0,7 \mathrm{~g} \mathrm{O}_{3} / \mathrm{g}$ DOC für Bezafibrat z. B. eine mittlere Entfernung von $70 \%$ erreicht, während für dieselbe Entfernung von Benzotriazol eine spezifische Ozondosis von $>0,9 \mathrm{~g} \mathrm{O}_{3} / \mathrm{g}$ DOC notwendig ist. Basierend auf den Ergebnissen wird eine spezifische Ozondosis von $0,7 \mathrm{~g} \mathrm{O}_{3} / \mathrm{g}$ DOC empfohlen, da hier die ozonaffinen Substanzen vollständig und die moderat affinen im Mittel zu etwa $60 \%$ entfernt werden, während die Bildung von Oxidationsnebenprodukten (Bromat) gering ist. Eine Erhöhung der spezifischen Ozondosis würde einen gegenüber den erzielten Effekten unverhältnismäßig höheren Betriebsmitteleinsatz erfordern. Mittlerweile wird jedoch vermehrt eine geringere Ozondosis unter $0,7 \mathrm{~g} \mathrm{O}_{3} / \mathrm{g}$ DOC diskutiert, da z.B. Hormone (v. a. Östrogene) und schnell reaktive Substanzen dadurch bereits beinahe vollständig entfernt werden. Die Dosierung muss sich letztendlich nach der gesetzlich geforderten Reinigungsleistung richten.

Für Überwachung bzw. Steuerung erwies sich die UV-Absorption als po- 
tenzieller Kandidat, da die Spurenstoffentfernung von moderat mit Ozon reagierenden Spurenstoffen gut mit der Reduktion des $\mathrm{SAK}_{254}$ korreliert. Weil Ozon selbst auch einen Adsorptionspeak bei etwa $254 \mathrm{~nm}$ aufweist, wird für eine Steuerung jedoch keine reine $\mathrm{SAK}_{254}$-Messung vorgeschlagen, die bei nur einer Wellenlänge $(254 \mathrm{~nm})$ erfolgt, sondern eine DOC-Modellierung über einen Wellenlängenbereich der UV/Vis-Spektroskopie zwischen 250 und $280 \mathrm{~nm}$ sowie einer Wellenlänge aus dem sichtbaren Spektrum zur Trübungskompensation. Die DOCLaborwerte können mit einem derartigen Modell sehr gut und statistisch ausreichend genau $\left(\mathrm{R}^{2}=0,91\right)$ abgebildet werden und stehen sofort zur Verwendung als neuralgischer Steuerungsparameter der Ozonanlage bereit. Ein Vorteil der DOC-Modellierung über eine spektrometrische UV/Vis-Messungen liegt in der Anpassung an einen breiten DOC-Bereich, um auch Mischwasserfälle (Verdünnung) abbilden $\mathrm{zu}$ können, was mit einem einfachen linearen $\mathrm{SAK}_{254}$-Modell nicht möglich ist.

Die Untersuchung von zwei Reaktorkonfigurationen zur Simulation eines voll durchmischten Reaktors bzw. eines kaskadierten Reaktors zeigte hingegen keine Unterschiede in der Spurenstoffentfernung oder $\mathrm{SAK}_{254}$-Reduktion. Für die bauliche Ausführung bedeutet dies, dass beide Reaktorbauweisen gleichgestellt werden können. Auch die Variation des Eintragsortes für das Ozon in einem kaskadierten Reaktor (Aufteilung auf alle Reaktoren vs. Eintrag nur im ersten oder im ersten und dritten Reaktor) hatte keinen Einfluss auf die erreichte Entfernung.

Die Aufenthaltszeit von rund $10 \mathrm{~min}$ in der Ozonung erwies sich in den durchgeführten Untersuchungen mehr als ausreichend für die Reaktion der Spurenstoffe und selbst eine Reduktion um 1/3 führte zu keiner Verringerung der Spurenstoffentfernung. Die bislang geltende Empfehlung von im Mittel 20 min Aufenthaltszeit im Reaktionsund Abklingbereich erweist sich somit als sehr großzügig bemessen und kann ohne Auswirkungen auf die Entfernungsleistung auf 15 min reduziert werden, v. a. wenn ein Multibarrierensystem ausgeführt wird.

In Hinblick auf die untersuchten Varianten der Nachbehandlungsstufen für die Ozonung (Nachklingvolumen, Anthrazitfilter, nachgeschalteter Aktivkoh-
le-Druckfilter) kann zusammengefasst werden, dass Anthrazitfilter und Nachklingvolumen idente Ergebnisse liefern, was Restozonentfernung, Spurenstoffentfernung und Keimreduktion betrifft. Der nachgeschaltete GAK-Filter führt jedoch $\mathrm{zu}$ einer weiteren, teilweise signifikanten, Spurenstoffreduktion. Dies bedeutet, dass in der Praxis die Verfahrenskombination mit einem nachgeschalteten Aktivkohlefilter empfohlen wird.

Die Aktivkohlefiltration - sowohl wie eben beschrieben, als eine der Ozonung nachgeschaltete Stufe, wie auch als eigenständige Aufbereitungstechnik zur Entfernung von organischen Spurenstoffen - erwies sich im Projekt jedenfalls als praxistaugliches und robust zu betreibendes Verfahren. Relevante Parameter für die Steuerung und Regelung der Aktivkohle-Druckfilter sind der Behälterdruck und der Volumenstrom. Der Behälterdruck erwies sich im Versuchsbetrieb als praktikabler Steuerparameter für die Rückspülung.

Ein entscheidendes Kriterium bei der Planung einer weitergehenden $\mathrm{Ab}$ wasserreinigungsstufe mittels Aktivkohlefiltration ist neben Produktqualität bzw. -eigenschaften der verwendeten granulierten Aktivkohle auch der erreichbare Durchsatz, gemessen in Bettvolumina. Das Eliminationspotenzial der untersuchten Spurenstoffe ist von der Adsorbierbarkeit der jeweiligen Substanz an Aktivkohle abhängig. Bei der Aktivkohlefiltration als eigenständige Aufbereitungstechnik zur Entfernung von organischen Spurenstoffen konnte festgestellt werden, dass die ausgewählten Indikatorsubstanzen für sehr gut bis gut adsorbierbare Stoffe über die gesamte Filterlaufzeit (ca. 10.000 Bettvolumina) im Mittel zu mehr als $85 \%$ entfernt wurden und die Elimination am Ende der Filterlaufzeit immer noch über $60 \%$ lag. Bei den Indikatorsubstanzen für mittel bis schlecht adsorbierbare Stoffe war ab ca. 2000 Bettvolumina ein Rückgang in der Eliminationsleistung zu verzeichnen und nach etwa 4000 Bettvolumina wurden bei diesen Substanzen erste erhöhte Ablaufkonzentrationen im Filter, d.h. die Ablaufkonzentration lag über der Zulaufkonzentration des jeweiligen Stoffes, festgestellt. Solche Desorptionsprozesse können in Vielstoffgemischen wie im Kläranlagenablauf auftreten, wo schlechter adsorbierbare Stoffe durch besser adsorbierbare verdrängt werden können. So ergeben sich in Abhän- gigkeit von der betrachteten Substanz sowie von dem jeweiligen Ausmaß der notwendigen Entfernung für die Aktivkohle-Druckfilter unterschiedlich lange Standzeiten. Betrachtet man die sehr gut bis gut adsorbierbaren Substanzen, so können bei diesen in Abhängigkeit vom notwendigen Mindesteliminationsgrad bis zum Filterdurchbruch deutlich höhere Standzeiten erreicht werden. Bei einem notwendigen Mindesteliminationspotenzial von $70 \%$ beträgt die Standzeit für sehr gut bis gut adsorbierbare Indikatorsubstanzen mehr als 10.000 Bettvolumina.

Ein weiteres entscheidendes Kriterium für die Bemessung von Aktivkohlefiltern ist die Kontaktzeit. Der Versuchsbetrieb hat gezeigt, dass es bei Verringerung der Kontaktzeit zu einer Reduktion der Spurenstoffelimination kommt. Daher ist grundsätzlich eine möglichst hohe Kontaktzeit anzustreben, diese ist jedoch aus betriebsund kostentechnischer Sicht auf ein sinnvolles Maß zu begrenzen. Beim Aktivkohlefilter, der parallel zur Ozonung betrieben wurde, lag die Aufenthaltszeit bei etwa $30 \mathrm{~min}$, während diese beim nachgeschalteten GAK-Filter bei etwas mehr als einem Drittel lag.

Im Vorfeld der Planung sollten Adsorptionsversuche in Form von Adsorptionsisothermen oder Filterschnelltests mit der zu behandelnden Abwassermatrix und der zum Einsatz kommenden Aktivkohle durchgeführt werden.

\section{Synopse}

Sowohl Aktivkohle als auch Ozonung haben ihre Stärken und Schwächen. Für einen effizienten Ressourceneinsatz und die Entwicklung sinnvoller Konzepte für eine weitergehende Reinigung ist es unabdingbar, verfahrenstechnische, umsetzungsorientierte und betriebliche Erfahrungen wissenschaftlich abgesichert $\mathrm{zu}$ erheben und für Variantenstudien bereitzustellen. Diesbezügliche Untersuchungen laufen zur Zeit im Rahmen des vom BMLFUW (Projektnummer B601389) geförderten Nachfolgeprojekts „KomOzAk II“.

Danksagung Das Projekt KomOzAk „Weitergehende Reinigung kommunaler Abwässer mit Ozon sowie Aktivkohle für die Entfernung organischer Spurenstoffe" wurde aus Mitteln des Bundesministeriums für Land- und Forstwirtschaft, Umwelt und Wasserwirtschaft (Projektnummern B202770 \& 100927) 
gefördert. Das Projekt wurde gemeinsam mit den Projektpartnern Donau Chemie AG, Messer Austria GmbH, VA TECH WABAG GmbH und Xylem Service $\mathrm{GmbH}$ durchgeführt. Ein spezieller Dank für deren Unterstützung sei an dieser Stelle auch an die begleitende Kläranlage gerichtet, an deren Standort die Versuchsanlage betrieben wurde.
Open access funding provided by TU Wien (TUW).

Open Access Dieser Artikel wird unter der Creative Commons Namensnennung 4.0 International Lizenz (http:// creativecommons.org/licenses/by/4. $0 /$ deed.de) veröffentlicht, welche die Nutzung, Vervielfältigung, Bearbeitung,
Verbreitung und Wiedergabe in jeglichem Medium und Format erlaubt, sofern Sie den/die ursprünglichen $\mathrm{Au}$ tor(en) und die Quelle ordnungsgemäß nennen, einen Link zur Creative Commons Lizenz beifügen und angeben, ob Änderungen vorgenommen wurden.

\section{Weiterführende Links}

Projektbericht KomOzAk: https://www.bmlfuw. gv.at/service/publikationen/wasser/KomOzAkLangfassung.html

Themenband „Spurenstoffe in der aquatischen Umwelt“ der Fachzeitschrift „Österreichische Wasser- und Abfallwirtschaft“ (ÖWAV); Springer
Verlag; Volume 65, Issue 5-6, Juni 2013 http:// link.springer.com/journal/506/65/5/page/1

ÖWAV-Positionspapier „Anthropogene Spurenstoffe in der aquatischen Umwelt“ (2013) http:// www.oewav.at/home/Service/Download - Positionspapiere
DWA-Themenband „Möglichkeiten der Elimination von anthropogenen Spurenstoffen - T 3/2015“ http://www.dwa.de

VSA-Plattform „Verfahrenstechnik Mikroverunreinigungen“" https://www.micropoll.ch 